\title{
LA REPOBLACIÓN FORESTAL PÚBLICA EN GALICIA DURANTE EL SIGLO XX
}

Eduardo Rico Boquete

Universidade de Santiago de Compostela 



\section{LOS ANTECEDENTES}

Las repoblaciones realizadas por los servicios forestales públicos en Galicia se remontan a comienzos del siglo XX, momento en el que los ingenieros destacados en los dos distritos forestales existentes (A Coruña-Pontevedra y Lugo-Ourense) llevaron a cabo las primeras plantaciones en áreas reducidas de determinados montes.

Ya en la década de 1920, los técnicos iniciaron las labores preparatorias de cara a la puesta en marcha de un ambicioso plan de repoblación, con el objetivo de conseguir un incremento de la producción de madera, y que tomó carta de naturaleza con la promulgación del R. Decreto-Ley de Repoblación de 26 de julio de 1926, que daría lugar a la formulación de un plan de actuación concreto para cada una de las provincias gallegas. Las labores efectuadas fueron, en todo caso, modestas, pues todo indica que no se superaron las 12000 hectáreas repobladas en el intervalo 1910-1936, destacando especialmente lo realizado en la provincia de Pontevedra ${ }^{1}$.

Dicha iniciativa venía a reforzar la acción reforestadora de carácter privado que se había puesto en marcha en la segunda mitad del siglo XIX, la cual había propiciado un incremento persistente y notable del área ocupada por el pino marítimo, especialmente en las zonas costeras y a lo largo de los principales valles fluviales, con el objetivo de satisfacer la demanda procedente de los sectores pesquero y agrícola (Rico Boquete 2014: 104-109). Por lo demás, esta progresión de las coníferas tomó un fuerte impulso desde la segunda mitad del siglo

\footnotetext{
1 Rico Boquete 1994: 104-128.
} 
XIX y fue un proceso paralelo al registrado en otros países europeos de influencia atlántica, especialmente a lo largo del siglo XX (Tsouvalis y Watkins 2000: 381-382; Whyte 2002: 170; Eggertsson et al. 2008: 24-26; Bunce et al. 2014: 8-9)².

Los propietarios de montes se habían dado cuenta de las excelentes cualidades que reunía la citada especie para la producción intensiva de un tipo de madera que se ajustaba muy bien a una demanda, nacional e internacional, que no dejaba de crecer y que les iba a permitir diversificar la producción, al tiempo que incrementaban sus ingresos. Por otra parte, esta expansión del pino gallego fue acompañada de un aumento del número de talleres de aserrío y del tráfico de madera, aserrada y en apeas, con destino al mercado nacional (zona mediterránea) e internacional (Gran Bretaña). De hecho, las estadísticas de comercio de madera, tanto las destinadas a la exportación como las que iban en régimen de cabotaje, atestiguan ese incremento de la actividad forestal. Y ello corrobora las informaciones proporcionadas por los técnicos y diversos publicistas, los cuales ya advertían de la creciente importancia que esa progresión del pino tenía en la economía de los labradores ${ }^{3}$.

En resumen, todo parece indicar que, durante la primera mitad del siglo XIX, las repoblaciones fueron muy modestas y el pino se introdujo lentamente en aquellos escenarios que le resultaron proclives, y e produjo una intensificación de la misma a partir de la década de 1890, en un contexto agrario caracterizado por el descenso de los precios agrícolas, producto de la crisis finisecular, el acceso de los campesinos a la propiedad plena, los apresamientos y el reparto de los montes comunales ${ }^{4}$.

2 Similares conclusiones se pueden ver en N. D. J. James (1981), R. Viney (1982) y A. Corvol-Dessert (1987).

3 Rico Boquete 2014: 104.

4 Balboa López 1990: 276-290; Artiaga Rego 2000: 465-466; Villares Paz 2000: 72-74; Bouhier 2001: 1068-1084; Soto Fernández 2006: 237-238. 


\section{LA REPOBLACIÓN FORESTAL DURANTE EL FRANQUISMO (1939-1975)}

La Orden de 21 de junio de 1938 instaba a la confección del Plan General de Repoblación Forestal de España con el objetivo de «llevar a la práctica uno de los puntos programáticos del Estado Nacional-Sindicalista» ${ }^{5}$. Poco tiempo después, la Ley de 8 de noviembre de 1939 daba el visto bueno al Plan extraordinario de trabajos forestales para los años 1939 y 1940 , con un presupuesto algo superior a los 34 millones de pesetas. En realidad se trataba de crear la masa forestal necesaria para garantizar la consecución de los objetivos estratégicos de la política forestal: «obtener primero la autarquía nacional en este aspecto y después ser exportadores» ${ }^{6}$. Por ello, los productos forestales también deberían de obtenerse «en indispensables condiciones de baratura y calidad a fin de que puedan competir en el mercado exterior» ${ }^{7}$.

En 1941, este objetivo autárquico se le inculcaba a los alumnos de la Escuela de Montes: «Hoy comenzamos a ver claro que es irremisible procurar que nuestra nación movilice sus dormidas energías y utilice sus recursos naturales hasta conseguir bastarse a sí misma». Y unos años después, en 1947, el director general de Montes aún consideraba «antisocial, antinacional, importar una sola tonelada de madera que el país pueda producir» ${ }^{8}$.

Posteriormente, cuando la realidad económica obligó a dejar de lado la arenga autárquica más intransigente, su lugar fue ocupado por el discurso sobre las posibilidades que se le abrían a España en el contexto de una Europa occidental deficitaria en productos forestales y de la que se aspiraba a formar parte, sin abandonar la pertinaz referencia al equilibrio de la balanza comercial con el exterior. En realidad, para esta mentalidad «ingenieril» todo parecía muy sencillo:

\footnotetext{
5 Concretamente, el punto XX del Programa de Falange Española.

6 Alarcón de la Lastra 1940: 3.

7 Alarcón de la Lastra 1940: 18.

8 Lleó Silvestre (1941: 17) y Robles Trueba (1947: 318), respectivamente.
} 
"Cuando haya madera suficiente y los precios sean más bajos, España podrá exportar, y ya figura la celulosa textil en algunos tratados comerciales como uno de los pocos productos industriales de exportación»?.

En todo caso, fue en aquel contexto de posguerra en el que se tomó la decisión de elaborar un plan general que permitiese, entre otras cosas, acometer con mayor intensidad la obra de la repoblación con el objetivo de incrementar la producción forestal y poder atender así las necesidades de materia prima en determinados sectores productivos. El citado plan, elaborado por los forestales Luis Ceballos y Joaquín Ximénez de Embún, contemplaba como objetivo fundamental la consecución de la autarquía en el sector forestal: «Hemos de acometer la tarea de investigar los montes que es preciso crear para conseguir con ellos, y conservando y aprovechando adecuadamente los que ya tenemos, cubrir las necesidades de España» ${ }^{10}$. Y para conseguir ese objetivo se consideraba imprescindible alcanzar los seis millones de hectáreas repobladas en 100 años.

No obstante, es conveniente señalar que los autores también aspiraban a generar beneficios para el conjunto de los ciudadanos; de hecho, afirmaban que el logro más importante consistiría en conseguir «no un máximo rendimiento en metálico, sino el máximo de bienestar para la población» ${ }^{11}$. En cierto modo, la propuesta de estos profesionales se inscribía dentro de lo que podríamos denominar «opción conservacionista», que tiene como objetivo la mezcla de especies, la creación de masas de distintas edades y caracteres y el aprovechamiento múltiple. En este sentido, resultaba óptima la utilización de los pinos, especialmente en aquellas zonas de suelos rasos y escasa vegetación en las que el empleo de las citadas coníferas permitiría asentar una masa forestal y crear las condiciones necesarias para, más adelante, seguir escalando las diferentes etapas hasta alcanzar la fase de vegetación clímax. Si bien, en este punto su fracaso fue absoluto,

\footnotetext{
9 Villegas de la Vega 1953: 20. Discurso también presente en Mesanza (1957) y Mesanza / Aspichueta (1968).

${ }^{10}$ Ceballos y Ximénez de Embún. Tomado de Ceballos 1996: 161.

${ }^{11}$ Ceballos y Ximénez de Embún. Tomado de Ceballos 1996: 210.
} 
pues la Administración no consideró oportuno aplicar tales principios a sus proyectos reforestadores e imprimió un carácter netamente productivista a su política forestal.

Asimismo, la intensificación de la repoblación fue una constante a lo largo de este período y aún se manifestó con más rotundidad en la década de 1950, coincidiendo con el acceso de Cavestany Anduaga al Ministerio de Agricultura, en un contexto de progresiva desaparición del entramado intervencionista del Estado y de avance de las propuestas de industrialización de las masas forestales que iba creando el Patrimonio Forestal del Estado (PFE). De hecho, en la reunión del Consejo de Dirección del PFE, el citado ministro instaba a sus componentes a que: «Dediquen todo su esfuerzo para imprimir a la actuación de este Organismo, la máxima intensidad en la labor repobladora, al objeto de crear la gran riqueza forestal que España necesita» ${ }^{12}$. Y el nuevo director del PFE, Paulino Martínez Hermosilla, anunciaba los objetivos de dicha institución: «En primer lugar, se ha previsto la realización, en un plazo que queremos que no exceda de 10 años, de un plan de repoblación de un millón de hectáreas» ${ }^{13}$.

Por otro lado, la Administración forestal era plenamente consciente de que para llevar a cabo las repoblaciones a gran escala que había previsto se encontraba con un importante obstáculo de carácter estructural, el cual era el escaso número y extensión de los montes propiedad del Estado. La solución vino de la recuperación de una figura contractual creada en 1926, fruto de la aplicación del R. Decreto-Ley de Repoblación Forestal de 26 de Julio del citado año, denominada consorcio de repoblación forestal. Estos fueron los primeros antecedentes de los consorcios establecidos por el Patrimonio Forestal y, posteriormente, por el Instituto para la Conservación de la Naturaleza (Icona).

\footnotetext{
12 Archivo de la Dirección General de Desarrollo Rural y Política Forestal (DGDRPF), Fondo documental del monte (FDM), Actas del Patrimonio Forestal del Estado, Acta de la sesión celebrada por el Pleno del Consejo del PFE, 26 de septiembre de 1951.

${ }^{13}$ Conferencia de prensa del director general de Montes y del Patrimonio Forestal del Estado, Excmo. Sr. Don Paulino Martínez Hermosilla, el día 24 de marzo de 1953. En Montes 50, 173-177.
} 
En el caso de Galicia, la Administración forestal estaba decidida a emplear las especies más aptas para la fabricación de aquellos productos considerados de primera necesidad (maderas, resinas, celulosa), siempre y cuando, como es obvio, lo permitiesen las condiciones del terreno. En este aspecto también existe una cierta continuidad, pues la Ley de creación del PFE, de 9 de octubre de 1935, establecía en su base cuarta que todas las repoblaciones deberían ser con especies de ciclo corto, criterio que fue modificado por el Decreto de 26 de agosto de 1939 al admitir la posibilidad de repoblar con toda clase de especies, aunque «dando la posible preferencia a las realizadas con especies de crecimiento rápido». De este modo, el Patrimonio Forestal no se vería limitado a repoblar solo en aquellas zonas aptas para sostener especies de crecimiento rápido, sino también hacerlo en toda la geografía española. Si bien, las zonas de más elevada producción maderera por hectárea y año estaban claras: «La cuenca del Bidasoa, Guipúzcoa y Vizcaya es zona de expansión del pino insignis; Santander y el oriente asturiano lo es del eucalipto y el occidente astur con toda la costa gallega lo es del pino pinaster ${ }^{14}$.

Ahora bien, y esto es útil recordarlo hoy en día, esta opción implicaba el rechazo total del objetivo formulado por Ceballos y Ximénez de Embún en su citado plan:

Parece lógico utilizar las ventajas que la región gallega nos ofrece para emprender en ella y con más ahínco y fervor la restauración de robledales y otras masas frondosas, utilizando los pinos, tan sólo, mientras suponga un medio para llegar a aquel fin. Es un error y un dolor toda protección que se haga en Galicia a la invasión de sus mejores suelos de monte, por especies que podrían vivir en cualquier parte cuyas masas se encuentran además a merced de una cerilla ${ }^{15}$.

\footnotetext{
${ }^{14}$ Echeverría 1940: 8.

${ }^{15}$ Ceballos y Ximénez de Embún. Tomado de Ceballos 1996: 272. Estos autores no desdeñaban el objetivo productivo, si bien debería ser una producción diversa y de calidad: «Tenemos una región gallega propia para castaños y robles, una región asturleonesa propia para robledales y hayedos, una región vascocántabra, de la que puede decirse otro tanto, una región pirenaica para el pino silvestre y el pino negro y el pinabete y el haya, todas ellas sin disputa regiones madereras, pura, lisa y esencialmente madereras». Ídem 1996: 237.
} 
De la intensidad y características del proceso nos hablan las cifras aportadas por el Patrimonio Forestal. En efecto, el total de repoblaciones logradas en Galicia, a 31 de diciembre de 1970, ascendía a 237237 ha. Las hectáreas trabajadas, incluyendo solo la primera repoblación, fueron 277 734; y si incluimos las segundas repoblaciones la superficie trabajada hasta 1970 ascendió a 305647 ha. Las estadísticas publicadas por el Patrimonio Forestal, en octubre de 1973, nos permiten analizar la distribución de especies utilizadas por el organismo en las plantaciones efectuadas en las tres décadas anteriores (vid. gráfico 1).

Gráfico 1. Evolución quinquenal de la repoblación y distribución de las especies empleadas en el período 1940-1970 (en hectáreas)

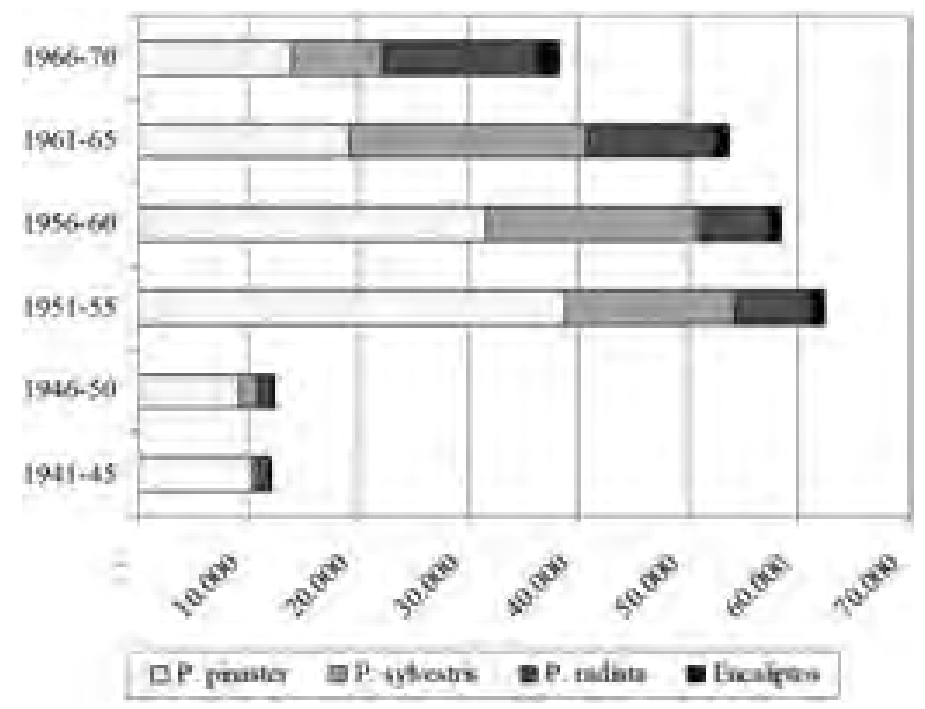

Fuente: Ministerio de Agricultura, Patrimonio Forestal del Estado. Inventario de repoblaciones en 31-12-1970, Madrid, 1973. Elaboración propia.

Como podemos constatar, aun con todas las reservas hacia los datos elaborados por la Administración forestal, la actividad repobladora mantuvo una clara tendencia ascendente que se hizo más notable a partir del año 1951, lo que supuso que en las dos décadas siguientes el promedio de repoblación anual se fijase en torno a las 10000 hectáreas, el doble que en la década de 1940. De 
hecho, la decisión del Instituto Nacional de Industria de implantar una factoría de celulosa en Pontevedra, el establecimiento de Tafisa en la misma ciudad o el proyecto de instalación de Papelera Española S.A., luego fracasado por la gran oposición suscitada, coincidieron en el tiempo con el auge de la actividad repobladora.

Asimismo, se observa un claro predominio del pinaster en las dos primeras décadas, la creciente presencia del pino radiata y también la del silvestre desde la década de 1950, momento en el que se intensificó la repoblación de las zonas del interior de Ourense y Lugo, y la todavía tímida presencia del eucalipto. En conjunto, y para las tres décadas referidas, destacamos la expansión del pinaster (123047 ha) y de los pinos silvestre (66 627 ha) y radiata (41 678 ha), además de 5802 ha de eucaliptos (vid. gráfico 2) ${ }^{16}$.

Gráfico 2. Especies empleadas en la repoblación, 1940-1970

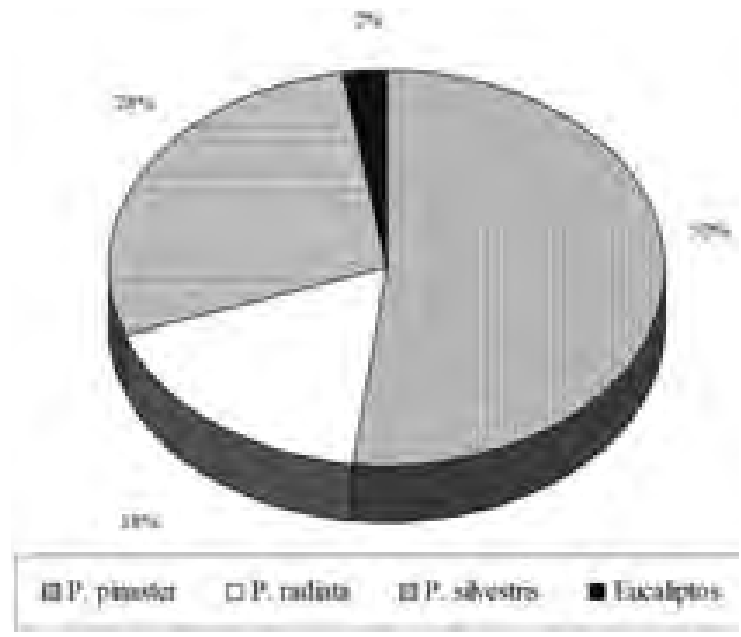

Fuente: Ministerio de Agricultura, Patrimonio Forestal del Estado. Inventario de repoblaciones en 31-12-1970, Madrid, 1973. Elaboración propia.

\footnotetext{
${ }^{16}$ La Sección de Celulosas del Instituto Forestal de Investigaciones y Experiencias (IFIE) había señalado en el año 1942 dos grupos de parcelas experimentales de producción, situadas en zonas pobladas con eucaliptos entre las extensas masas de pinar creadas por la Diputación en 1927.
} 
En el ámbito de las actuaciones forestales públicas, esta distribución de especies empleadas parece que empieza a cambiar a partir de la década de 1970, momento a partir del cual se aprecia un modesto pero paulatino incremento del empleo del eucalipto. Lo más probable, no obstante, es que, al igual que ocurrió en el siglo XIX con el pinaster, el uso del eucalipto ya estuviese más extendido en las repoblaciones efectuadas por los particulares. En todo caso, en Galicia tenemos constancia del uso de dicha especie en las repoblaciones efectuadas por Sniace, a finales de la década de 1950, en montes públicos y privados situados en los ayuntamientos de Cotobade, Caldas de Reis, Cuntis y Tomiño ${ }^{17}$. Por su parte, el Patrimonio Forestal también llevó a cabo repoblaciones con eucalyptus globulus en A Coruña y Pontevedra, y empleó otras variedades de eucaliptos en Ourense, quedando excluida inicialmente la provincia de Lugo.

En resumen, el predominio de las coníferas y el rechazo a la utilización de las frondosas autóctonas, aun en pequeñas parcelas y en contra del criterio que habían formulado los ingenieros Joaquín Ximénez de Embún y Luis Ceballos, supuso el triunfo definitivo de una orientación productivista que se impuso desde el primer momento en que comenzó la actividad del Patrimonio Forestal, renunciando a la constitución de masas forestales de mejor calidad y mayor valor añadido.

\section{LA REPOBLACIÓN DURANTE LA TRANSICIÓN Y EN EL MARCO DEL MODELO AUTONÓMICO}

Ahora bien, la desaparición del Patrimonio Forestal y su sustitución por el Instituto para la Conservación de la Naturaleza, en 1973, tampoco parece que haya contribuido a modificar determinadas directrices que estaban muy arraigadas en el quehacer de la Administración forestal.

\footnotetext{
${ }^{17}$ Por cierto, dichas repoblaciones también fueron impulsadas por D. Paulino Martínez Hermosilla, pero obrando ya en calidad de subdirector de Sniace. Un ejemplo de puertas giratorias que fue muy habitual en la época (Groome 1990).
} 
Dicho en otros términos, si la actuación del Patrimonio en Galicia estaba orientada, exclusivamente, a la creación de montes productores de madera, la primera de las leyes de repoblación forestal aprobada después del fin de la Dictadura venía a incidir en el mismo objetivo, lo que ya quedaba muy claro en el propio título de la misma. En efecto, la Ley de Fomento de la Producción Forestal, de 4 de enero de 1977, tenía como finalidad fomentar nuevas plantaciones de arbolado, "primordialmente con especies de rápido crecimiento», con el objetivo de conseguir un incremento sustancial de la oferta de madera ${ }^{18}$.

En el contexto de los grandes cambios políticos que estaban ocurriendo en el país y en medio de una profunda crisis económica, el incremento de la producción de madera para las industrias consumidoras se esgrimía como un factor que podría contribuir al equilibrio de la balanza comercial, al limitar las importaciones de madera o sus derivados y facilitar la competitividad de nuestros productos elaborados destinados a la exportación, por ejemplo tableros y celulosa.

Por ello, la nueva legislación tenía como misión fundamental prestar la ayuda necesaria para realizar plantaciones que permitiesen obtener «en el plazo más breve posible» importantes cosechas de madera, priorizando la plantación de especies de crecimiento rápido así como la intervención en masas arboladas con medidas selvícolas que fuesen susceptibles de generar un incremento en la oferta de madera. Objetivo urgente al que también debían de contribuir aquellas masas forestales que, aun teniendo carácter protector, pudiesen aportar cuantiosos recursos maderables.

En principio, tales ayudas irían destinadas a los propietarios y a los usuarios de la madera; es decir, también se trataba de fomentar la participación de la iniciativa privada en un momento en el que la industria consumidora «tenía un gran interés en contar con la materia prima necesaria». Además de toda una serie de beneficios fiscales, las ayudas más importantes consistían en subvenciones y créditos oficiales; aquellas podían alcanzar hasta un máximo del $50 \%$ del

${ }^{18}$ Boletin Oficial del Estado, no 7, de 8 de enero de 1977, 361-364. 
presupuesto total y estos en concepto de anticipo reintegrable. En conjunto, estas ayudas podrían alcanzar el $95 \%$ del presupuesto de la obra.

Las preferencias establecidas en la Ley abundaban en ese carácter productivista de la misma, determinando que serían clasificados como preferentes los créditos aplicados en montes poblados con «especies de gran rendimiento en madera» así como aquellos predios en los que las masas creadas fuesen regulares, estuviesen sometidas a cortas a echo y que la corta final se realizase en el plazo de diez años. Ciertamente, con estos criterios no era necesario especificar el nombre de las especies consideradas más interesantes. La Administración pública optaba claramente por dar un gran impulso a la repoblación con eucaliptos; como refiere la propia Ley en la exposición de motivos, se trataba de solucionar «[1]a creciente demanda de madera por parte de la industria y la necesidad de subvenir a la oferta para lograr que cubra las necesidades de aquélla». De nuevo, tal y como había sucedido con las leyes de repoblación de 1926 y de creación del Patrimonio Forestal, la «escasez» de madera para la industria se convertía en la justificación principal que daba impulso a la repoblación, mejor dicho, a la plantación con especies de crecimiento rápido.

Por último, pero igual de importante, al menos en el caso de Galicia, la aplicación de los beneficios previstos en la Ley tenía carácter prioritario en el caso de los montes afectados por incendios, lo cual, en cierta medida, significaba jugar con fuego si tenemos en cuenta la cantidad y magnitud de los incendios producidos en aquellos años (119634 ha en 1978). Aunque, como es obvio, no todas las superficies fuesen aptas para ser ocupadas con eucalipto, no cabe duda de que tales ayudas podían constituir una tentación en caso de que los propietarios quisiesen llevar a cabo un cambio de especie en sus predios.

Al mismo tiempo, también es necesario reconocer que la nueva legislación ofrecía a las comunidades y propietarios particulares mejores condiciones económicas para proceder a la repoblación de sus montes, incluyendo la posibilidad de modificar los antiguos consorcios y transformarlos en convenios, como así se acordó en múltiples casos. 
Sin embargo, a la hora de analizar los resultados de las actuaciones forestales públicas durante el último cuarto del siglo XX, nos encontramos, aunque parezca paradójico, con ciertos problemas derivados de la inexistencia de datos estadísticos para varios años (de 1988 a 1991, 1995, 1997). Y, cuando estos existen, son tan parcos que resulta casi imposible saber, por ejemplo, qué especies se utilizaron en dichas plantaciones, obligando al investigador a acudir a vías indirectas y menos fiables para saber algo más sobre las repoblaciones.

En un primer momento, y en términos cuantitativos, la actuación repobladora de la Administración pública se mantuvo en una media anual similar a la registrada en la última década de la Dictadura; de hecho, para el período 1966-1975 la media de hectáreas repobladas fueron 6859, mientras que en la década siguiente, 1976-1986, la media obtenida se cifró en 7948 ha, algo superior a la de la etapa anterior. Por supuesto, y dado que en la estadística se incluyen primeras y segundas repoblaciones, la superficie realmente ocupada es menor que la resultante de la suma de aquellas. O dicho en otros términos, las cifras siempre se refieren al número de hectáreas trabajadas, por cuanto constituyen el mejor indicador para conocer la intensidad del proceso y revelan el verdadero objetivo de la actuación reforestadora. Esa persistencia del esfuerzo repoblador se observa con claridad en el gráfico que nos muestra la evolución anual de las plantaciones (vid. gráfico 3).

Por otro lado, la ya señalada ausencia de datos para determinados años dificulta la comparación con lo sucedido en la última década del siglo XX; no obstante, las cifras manejadas no indican un cambio sustancial en la intensidad del proceso, como podemos advertir en el gráfico que recoge el total acumulado (vid. gráfico 4).

Al mismo tiempo, la Administración también puso en marcha un programa de ayuda a los propietarios para realizar plantaciones con especies de crecimiento rápido en sus propiedades, incluyendo en dicho plan a todas aquellas empresas que poseyesen montes en Galicia, sobre todo las directamente vinculadas al sector forestal. De ese modo, entidades como Proullasa, Aguas de Mondariz, 
Gráfico 3. Repoblación anual, 1941-1999 (en ha)

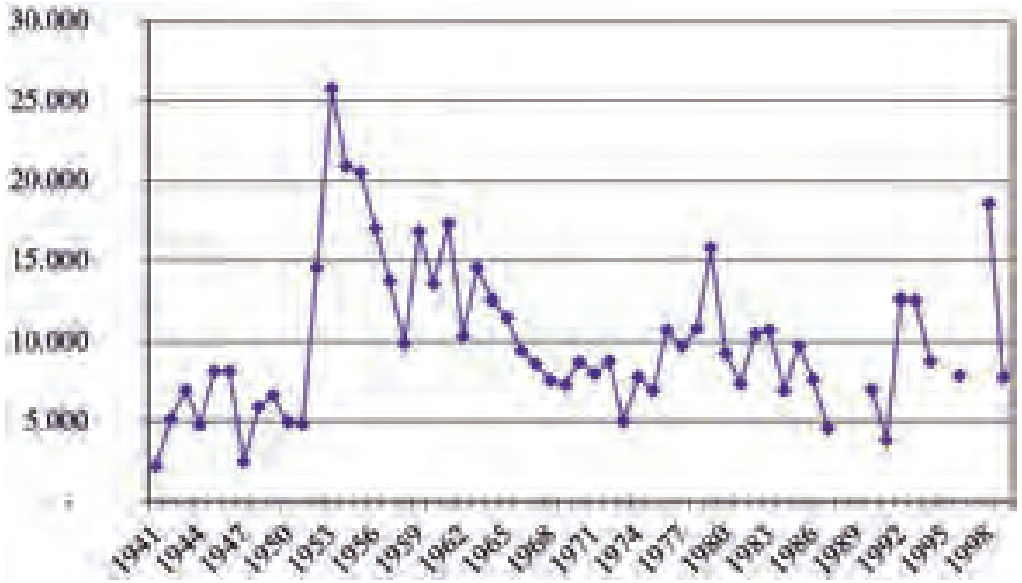

Fuente: Patrimonio Forestal del Estado. Memoria-Resumen de los trabajos realizados en el decenio 1940-1949; Patrimonio Forestal del Estado. Memoria-Resumen de los trabajos realizados en el cuatrienio 1950-53; Estadística forestal de España, 1946-1971; Anuario de Estadistica Agraria, 1972-1990.

Gráfico 4. Repoblación forestal, 1940-1999. Total acumulado (en ha)

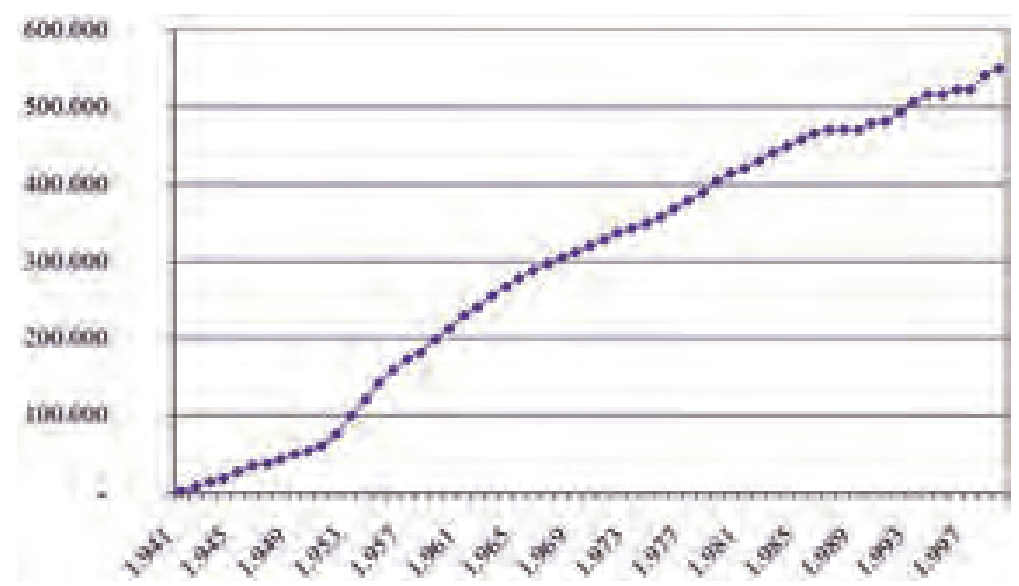

Fuente: Patrimonio Forestal del Estado. Memoria-Resumen de los trabajos realizados en el decenio 1940-1949; Patrimonio Forestal del Estado. Memoria-Resumen de los trabajos realizados en el cuatrienio 1950-53; Estadística forestal de España, 1946-1971; Anuario de Estadística Agraria, 1972-1990. 
Sniace o Ence pudieron acogerse al mismo. En todo caso, con o sin ayudas públicas, lo que parece evidente es que tuvo que darse un incremento sustancial de las repoblaciones de eucalipto llevadas a cabo por propietarios particulares, aunque en este aspecto la deficiencia de las estadísticas no permite traducirlo a cifras. No obstante, y aunque de manera indirecta, podemos constatar esa expansión del eucalipto, incentivada por el incremento de la demanda procedente de las grandes industrias consumidoras (Ence, Sniace, Ceasa), junto con la retracción de otras especies.

De hecho, si comparamos la evolución de las cortas en el largo plazo, podemos apreciar la tendencia creciente del eucalipto, que acaba alcanzando a las coníferas (vid. gráfico 5). Lo anterior no sería más que la lógica consecuencia de las intensas repoblaciones con eucalipto realizadas en las últimas décadas del siglo XX, al amparo de la Ley de Producción Forestal de 1977. En conclusión, esta evolución de las cortas de madera confirma el mayor peso que fue tomando el eucalipto y el retroceso de las coníferas en general, lo que implica un claro cambio de tendencia en relación con lo sucedido en el período anterior, de absoluto predominio del pino gallego en las labores de repoblación.

Gráfico 5. Cortas de madera de coníferas y eucaliptos, 1946-1999 (en m³ c/c)

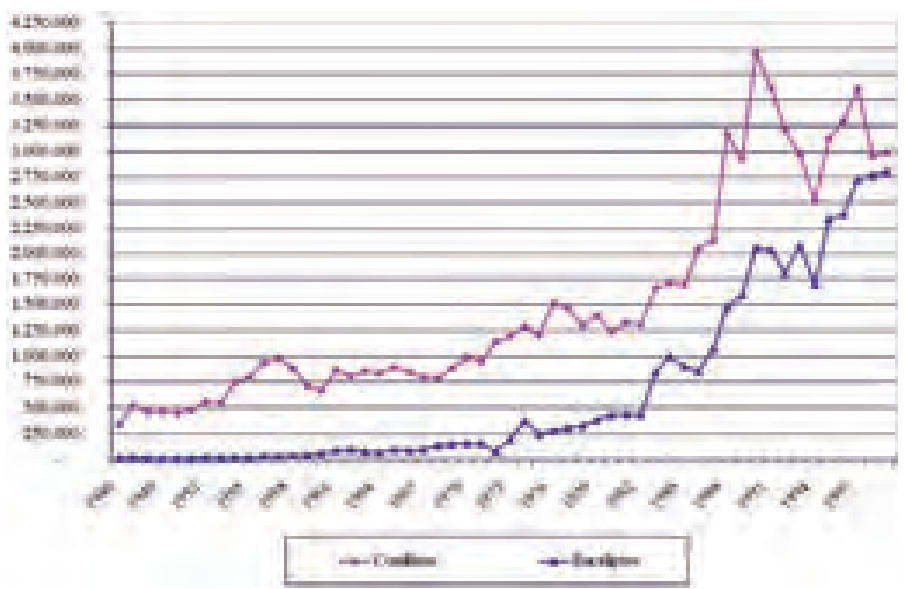

Fuente: Estadistica forestal de España, 1946-1971; Anuario de Estadistica Agraria, 1972-1990; Anuario de Estadistica Agraria, 1991-1999. 


\section{REFERENCIAS BIBLIOGRÁFICAS}

Abelló de la Torre, M. A. (1988): Historia y evolución de las repoblaciones forestales en España, Madrid, Universidad Complutense.

ALARCÓN DE LA LASTRA, Ricardo (1940): El triunfo nacional y su repercusión en las orientaciones de la industria y el comercio, Madrid, Ministerio de Agricultura.

Artiaga Rego, Aurora (2000): «A renda foral en Galicia a fins do século XIX», en Lourenzo Fernández Prieto (ed.), Terra e progreso. Historia Agraria da Galicia contemporánea, Vigo, Xerais, 443-470.

Balboa López, Xesús (1990): O monte en Galicia, Vigo, Xerais.

BOUHier, Abel (2001): Galicia. Ensaio xeográfico de análise e interpretación dun vello complexo agrario, Benxamín Casal Vila (trad.), Santiago de Compostela, Consellería de Agricultura, Gandería e Política Agroalimentaria / Caixanova.

Bunce, Robert G. H. / Claire M. Wood / Simon M. SMART / Rachel OAKLEY / Gareth Browning / Mike J. Daniels / Philip Ashmole / John Crosswell / Kate Holl (2014): «The Lanscape Ecological Impact of Afforestation on the British Uplands and some initiatives to restore native woodland cover», Journal of Landscape Ecology, 7:2, 6-24.

Cavestany, Rafael (1958): Una politica agraria. Discursos, Madrid, Dirección General de Coordinación, Crédito y Capacitación Agraria del Ministerio de Agricultura.

Ceballos Fernández de Córdoba, Luis (1996): Homenaje en su centenario. Tres trabajos forestales, Madrid, Parques Nacionales.

Corvol, Andrée (1987): L'Homme aux Bois. Histoire des relations de l'homme et de la fôret (XVII-XX siècle), París, Fayard.

ECHEVERRÍA BALlarín, Ignacio (1940): «Il rimboschimento del nord della Spagna e l'autarchia cellulosica», La Rivista Forestale Italiana, extracto dos números 8-9.

Eggertsson, Olafur / Per Hola Nygaard / Jens Peter SkovsgaArd (2008): «History of afforestation in the Nordic countries", en Gudmundur Halldorsson / Edda Sigurdis Oddsdottir / Bjarni Didrik Sigurdsson (eds.), Affornord. Effects on Ecosystems, Landscape and Rural Development, Dinamarca, Nordic Council of Ministers.

Gómez Mendoza, Josefina / Rafael Mata Olmo (1992): «Actuaciones forestales públicas desde 1940. Objetivos criterios y resultados», Agricultura y Sociedad, 65, 15-64.

Groome, Helen (1990): Historia de la politica forestal en el Estado español, Comunidad de Madrid, Agencia de Medio Ambiente.

JAMEs, Noel D. G. (1990): A History of English Forestry, Oxford, Basil Blackwell.

JimÉnEZ BlAnCO, José Ignacio (2002): «El monte: una atalaya de la Historia», Historia Agraria, 26, 141-190.

Lleó Silvestre, Antonio (1941): Enseñanzas sociales del bosque. Inauguración en la Escuela Especial de Ingenieros de Montes del curso 1940-41 y Homenaje a los Ingenieros y Alumnos... caidos por Dios y por España, Madrid, Dirección General de Montes, Caza y Pesca fluvial.

Martínez Hermosilla, Paulino (1990): «Enfoque histórico de los trabajos de restauración», Ecología, 1, 367-372.

MESANZA Ruiz DE SALAS, Bernardo de (1957): «Una aportación a la Unión Europea: la cornisa cantábrica repoblada», Montes, 78, 395-396.

MESANZA, Bernardo de / Ignacio Aspichueta (1968): «España puede y debe ser la reserva forestal que necesita la pequeña Europa», Montes, 139, 51-52. 
Ministerio de Agricultura (1951): Patrimonio Forestal del Estado. Memoria-Resumen de los trabajos realizados en el decenio 1940-1949, Madrid.

Ministerio de Agricultura (1954): Patrimonio Forestal del Estado. Memoria-Resumen de los trabajos realizados en el cuatrienio 1950-1953, Madrid.

Montero De Burgos, José Luis (1990): «Evolución vegetal. Óptimo natural y óptimo forestal», Ecología, Número extraordinario, 1, 309-320.

OоsтHовк, K. Jan (2013): Conquering the Highlands: A history of the afforestation of the Scottish uplands, ANU Press, Acton.

OrtuÑo Medina, Francisco (1974): «La repoblación forestal inversión promotora de la industria en España", Montes, 177, 281-286.

Ortuño Medina, Francisco (1975): «Consideraciones sobre la política forestal en España y su relación con la protección del medio ambiente», Montes, 181, 209-221.

Rico Boquete, E. (1995): Politica forestal e repoboacións en Galicia, 1941-1971, Santiago de Compostela, Universidade de Santiago de Compostela.

Rico Boquete, E. (2000): «Política forestal y conflictividad social en el Noroeste de España durante el Primer Franquismo, 1939-1959», Historia Social, 38, 117-140.

Robles Trueba, Salvador (1947): «La ordenación de montes, principio político», Montes, 16, 317-320.

SOTO FERnÁNDEZ, David (2006): Historia dunha agricultura sustentábel. Transformacións produtivas na agricultura galega contemporánea, Santiago de Compostela, Xunta de Galicia, Consellería do Medio Rural.

Tsouvalis, Judith / Charles WatKINS (2000): «Imagining and creating forests in Britain, 1890-1939», en M. Agnoletti / S. Anderson (eds.), Forest History: International Studies on Socio-economic and Forest Ecosystem Change. Report no 2 of the IUFRO Task Force on Environmental Change, Nova York, CABI en asociación con IUFRO, 371-386.

VINEY, Raymond (1982): «La progressión des résineux en Bourgogne depuis la fin du XVIII ème siècle», en Actes du Symposium International d'Histoire Forestière, 24-28 septembre 1979, Nancy, École Nationale du Génie Rural, des Eaux et des Forêts, 2 vols.

VILLARES PAZ, Ramón (2000): «A agricultura galega, 1870-1930. Unha época de grandes transformacións», en Lourenzo Fernández Prieto (coord.), Terra e progreso. Historia Agraria da Galicia contemporánea, Vigo, Xerais, 61-82.

VILlegas DE LA VeGa, Roberto (1953): Repoblaciones de eucalipto y pino insigne en el Norte de España, Madrid, Escuela Especial de Ingenieros de Montes.

WATKINS, Ch. (ed.) (1998): European Woods and Forests: Studies in Cultural History, Wallingford, (Oxon), $\mathrm{CAB}$ International.

WHYTE, Ian D. (2002): Landscape and History since 1500, London, Reaktion Books ltd. 